la numération des germes microbiens. Le Lait, 1938, 18, 225. - II. Sur la colimétrie appliquée aux contrôles de la pasteurisation des laits pas. teurisés. Le Lait, 1939, 19, 113.

G. Guittonneau et R. Chevalier. Nouvelles techniques de préparation d'un milieu de culture à base de lait digéré ; le lait digéré par la papaïne. Le Lait, 1941, 21, 1.

Tanner. Microbiology of Foods. 2nd édition. Garrard Press, Champaign, Illinois, 1944, 320-334 / Bacterial Count of milk (bibliographie importante sur la numération des germes de toute nature).

G. Thieulin et R. Vuirladume. Eléments pratiques d'analyse et d'inspection

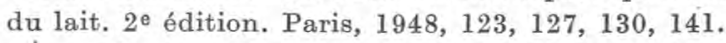

American Public Health Association. Standard methods for the examination of dairy products, 9th édition, New York, 1948.

S. -B. Thomas et J. M. Harcombe. Determination of the Coli-aerogenes content of milk by plating methods. Dairy Science Abstracts, 1954, 16, $n^{\circ} 8,608$. (Bibliographie importante sur le dénombrement des coliformes par cultures en milieux solides.)

G. Thieulin et D. Basille. Bul. Acad. Vet., 1955, n 7 .

\title{
DÉTECTION DES LAITS RECONSTITUÉS ET CHOIX D'UN RÉVÉLATEUR
}

\author{
par \\ V. TOUBOL \\ Ingénieur Chimiste I.C.N. \\ Directeur du laboratoire officiel de chimie de Casablanca \\ (Service de la Répression des Fraudes)
}

Les conditions économiques au Maroc ont suscité un intérêt exceptionnel dans la pratique d'une fraude simple à réaliser et pratiquement impossible à déceler.

Il s'agit de la substitution au lait frais d'un lait reconstitué à partir de lait en poudre écrémé, produit dont l'importation était tolérée pour la fabrication des aliments du bétail.

Si l'écrémage pouvait être décelé dans le cas d'une forte substitution, il n'en était pas de même pour une substitution de l'ordre de $20 \%$, car le lait des vaches marocaines a une teneur en matière grasse relativement élevée.

Voici quelques résultats authentiques sur plusieurs étables (déterminations faites au Laboratoire Officiel de Chimie, Service de la Répression des fraudes, en 1953) :

Matière grasse (en grammes par litre), de laits de mélange provenant d'étables différentes ou à différentes époques : $39,36,52$, $47,37,31,43,37,39,35,40,46,35,44,40,38,43,46,40,43,38$, 
$39,38,40,38,40,37,37,41,41,40,38,41,44,33,38,40,40,39$, $40,47,37$.

Sur les 42 laits de mélange examinés : 2 sont inférieurs ou égaux à $33 ; 18$ sont compris entre 33 et $40 ; 22$ soit plus de $50 \%$ vont de 40 à 52 grammes par litre.

Par contre, la substitution de $20 \%$ de lait valant 15 franes le litre à du lait frais valant 75 francs, permet un bénéfice assez substantiel pour tenter un fraudeur. Il fallait donc mettre fin à cette situation.

Certains auteurs ont essayé de résoudre le problème par l'étude du pouvoir réducteur des protéines. Ce pouvoir est en accroissement pour les protéines du lait ayant subi l'action de la chaleur (cas du lait en poudre). (L. Clay, M. BURke et S. Junker : Journal Of. Assoc. Off. Agric. Chem., 1955, no 2, p. 310-318.)

Mais cet accroissement qui a été étudié pour des laits de provenance américaine par les auteurs de la méthode, est-il valable pour les laits d'autres pays?

De plus, cet accroissement est extrêmement variable. Il ne semble pas que l'on puisse mottre en évidence, une substitution de tout ou partie de lait frais par du lait en poudre en se basant sur cet accroissement, ce pouvoir étant lui-même inconstant. En effet, sur 411 échantillons examinés, provenant de six Etats différents, les auteurs constatent que le pouvoir réducteur des protéines va de 0,90 à $4,18 \%$, soit des variations de l'ordre de $3 \%$.

Par ailleurs, sur 45 échantillons ayant reçu $10 \%$ de lait reconstitué, l'accroissement de ce pouvoir réducteur est toujours inférieur à $3 \%$ sauf dans cinq cas.

Ainsi, sans parler des difficultés qui interviennent du fait que l'échantillon ne doit avoir subi aucune réfrigération ni addition d'antiseptique, que la détermination de ce pouvoir réducteur nécessite un matériel spécialisé et coûteux à la portée seulement d'un petit nombre de laboratoires, la méthode ne peut pas permettre de déceler avec certitude la tromperie.

D'autres auteurs (VALLIER, Le Lait, 1955, noo 347, 372) ont mis à profit la modification que subit la caséine pour des raisons encore mal éclaircies et qui, dans le lait reconstitué, la rend insoluble par la méthode d'Adam-Mellière.

Pratiquement, cette insolubilisation ne peut se vérifier que si le lait n'a été additionné d'aucun antiseptique et particulièrement le formol ou son polymère, le trioxyméthylène. Le temps qui s'écoule depuis la traite peut même apporter un trouble dans les résultats. Nos essais nous ont montré qu'il en était de même avec un lait pasteurisé. 
Ces faits ne sont pas compatibles avec le fonctionnement normal du service de la répression des fraudes.

Dès lors, nous avons pensé qu'il serait plus efficace d'adopter l'artifice du révélateur et le choix de celui-ci n'était pas exempt d'écueils.

S'agissant d'un produit pouvant être frauduleusement introduit dans l'alimentation des nourrissons, le choix des révélateurs était limité.

Nous avons proposé l'amidon qui, tout d'abord, a paru être suspect aux services de santé, mais qui fut admis par la suite.

L'amidon, pour remplir le rôle que nous attendions, devait pouvoir, à froid, dans les conditions de solubilisation de la poudre, entrer aussi dans la solution. Il ne pouvait done s'agir que d'amidon soluble à froid.

De nombreuses difficultés ont été rencontrées pour obtenir des fabrieants l'utilisation d'un amidon répondant à ces conditions.

Ces difficultés provenaient de ce que l'amidon employé était insuffisamment transformé et se retrouvait au fond de la dissolution après quelques instants de repos.

Le choix de la matière première, qui semble être la fécule de pomme de terre, et la température de transformation, ont $\mathrm{du}$, cependant, être mis au point. Il n'y a plus à présent, de difficulté et le révélateur ainsi introduit par le fabricant, répond intégralement aux qualités que l'on attend : sensibilité, détection facile, inocuité.

Le procédé d'introduction de l'amidon reste secret, mais nous avons de bonnes raisons de penser que l'amidon est traité avec le lait dans l'atomiseur pour obtenir l'homogénéité aussi parfaite que celle que nous avons constatée.

Ainsi, la poudre de lait écrémé peut se trouver dans le commerce à condition de renfermer 5 grammes d'amidon soluble à froid, par kilogramme.

Pour vérifier la présence d'amidon dans la poudre, il suffit d'en faire à froid, une solution aqueuse au dixième, et d'essayer le réactif iodo-ioduré. Une seule goutte de ce réactif pour 10 centimètres cubes de solution, fait apparaître une teinte bleu violacée intense, colorant le liquide dans son intégralité. Au contraire, si l'amidon n'était pas soluble, il n'y aurait aucune coloration apparente mais, après quelques instants de repos, on verrait se rassembler un petit dépôt noirâtre. L'amidon en cet état ne saurait donner satisfaction.

Le contrôle des laits frais se trouve dès lors facilité : il suffira d'une ou deux gouttes de réactif iodo-ioduré, pour obtenir la teinte 
caractéristique dans un produit contenant $10 \%$ et plus de lait reconstitué.

La coloration bleue obtenue dans un essai positif disparaît au bout d'un certain temps, mais une nouvelle goutte de réactif la fait réapparaître aussi intense.

Il est possible aussi de révéler la présence de lait reconstitué dans les produits lactés, cacaotés ou teintés. Ces produits seront d'abord soumis à une défécation au moyen de l'acétate de zinc et du ferrocyanure, puis, sur le liquide filtré, incolore, la réaction à l'iode restera positive s'il s'agit de lait reconstitué à partir de poudre contenant le révélateur.

\title{
LAIT PASTEURISÉ - LAIT STÉRILISÉ
}

II

\author{
Aspects techniques et hygiéniques \\ du problème des laits destinés à la consommation \\ par \\ G. MOCQUOT \\ (Fin.)
}

DEUXIEME PARTIE

\section{DESTRUCTION DES MICROBES DE LA FLORE BANALE AU COURS DE LA PASTEURISATION ET QUALITÉ DE CONSERVATION DU LAIT PASTEURISÉ}

La pasteurisation détruit les bactéries pathogènes, mais elle améliore également les qualités de conservation du lait. Du point de vue technique et commercial, ce fait est très important, car il permet de disposer du temps nécessaire au transport et à la distribution du lait pasteurisé. Il est difficile de concevoir comment l'alimentation en lait des grands centres urbains pourrait, de nos jours, être assurée convenablement sans la pasteurisation.

En fait, un seul pays, la Suisse, alimente ses villes en lait cru pour la plus large part. Mais les conditions de production et de récolte du lait, dans un rayon de ramassage relativement restreint autour des centres urbains qui n'atteignent pas les dimensions des grandes capitales, y sont tout à fait particulières et n'ont que peu ou pas leur équivalent dans les autres pays d'Europe ou d'Amérique.

Un lait pasteurisé, dans les conditions définies plus haut pour la destruction des bactéries pathogènes, possède une flore lactique plus pure que celle du lait cru, c'est-à-dire que la proportion des 